\title{
A Luminescent NOTA-based Terbium(III) 'Turn-Off' Sensor for Copper
}

Michèle Clerc, ${ }^{[a]}$ Franz Heinemann,,${ }^{[a, b]}$ Bernhard Spingler, ${ }^{[a]^{*}}$ and Gilles Gasser ${ }^{[\mathrm{b}]^{*}}$

[a] M. Clerc, F. Heinemann, Prof. Dr. B. Spingler

Department of Chemistry, University of Zurich

Winterthurerstrasse 190, CH-8057, Zurich, Switzerland

E-mail: spingler@chem.uzh.ch

[b] F. Heinemann, Dr. G. Gasser

Chimie ParisTech, PSL University, Laboratory for Inorganic Chemical Biology

F-75005 Paris, France

E-mail: gilles.gasser@chimieparistech.psl.eu

WWW: www.gassergroup.com 


\section{SUPPORTING INFORMATION}

\section{Contents}

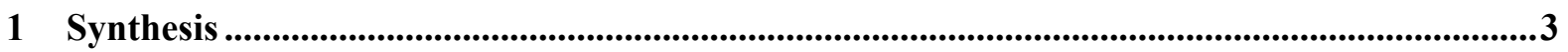

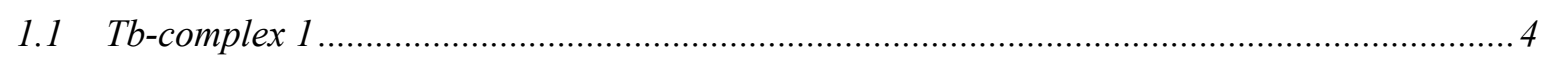

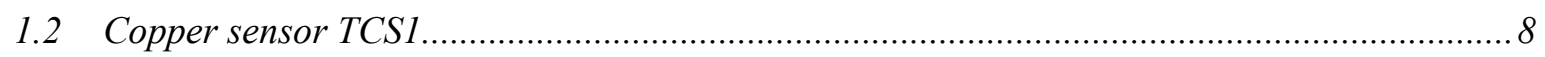

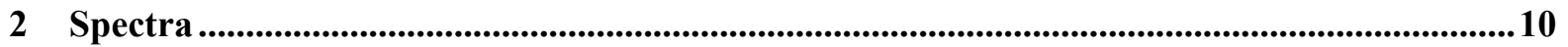

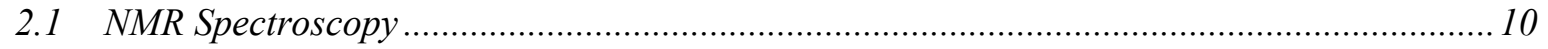

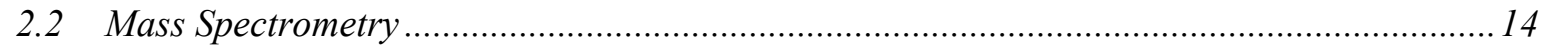

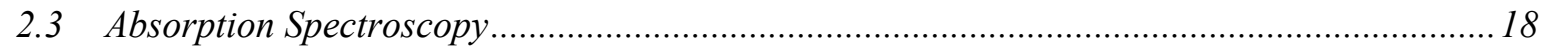

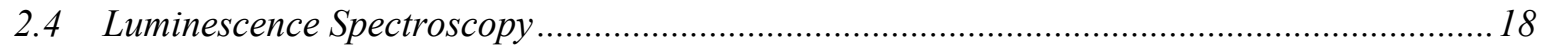

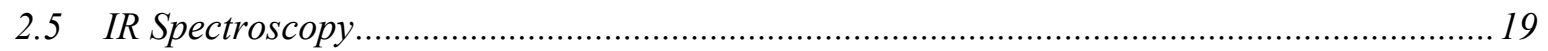

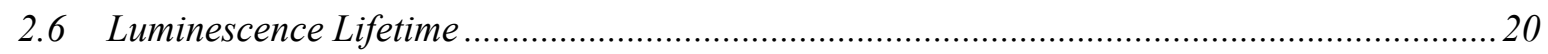

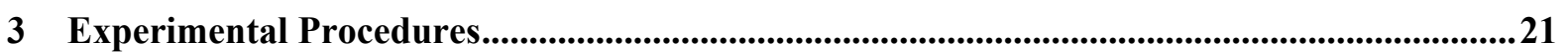

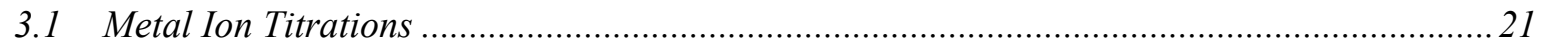

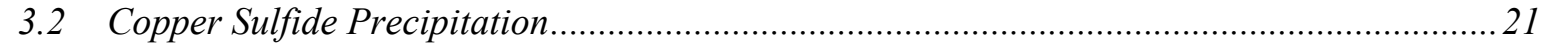

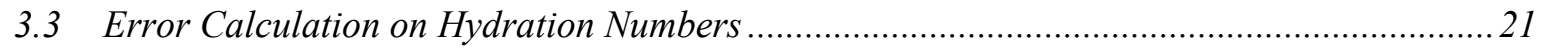

4 Detection Range and LOD of Small Molecule $\mathrm{Cu}^{2+}$ Sensors..................................................22

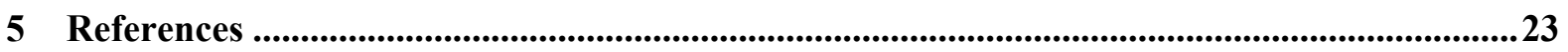




\section{Synthesis}

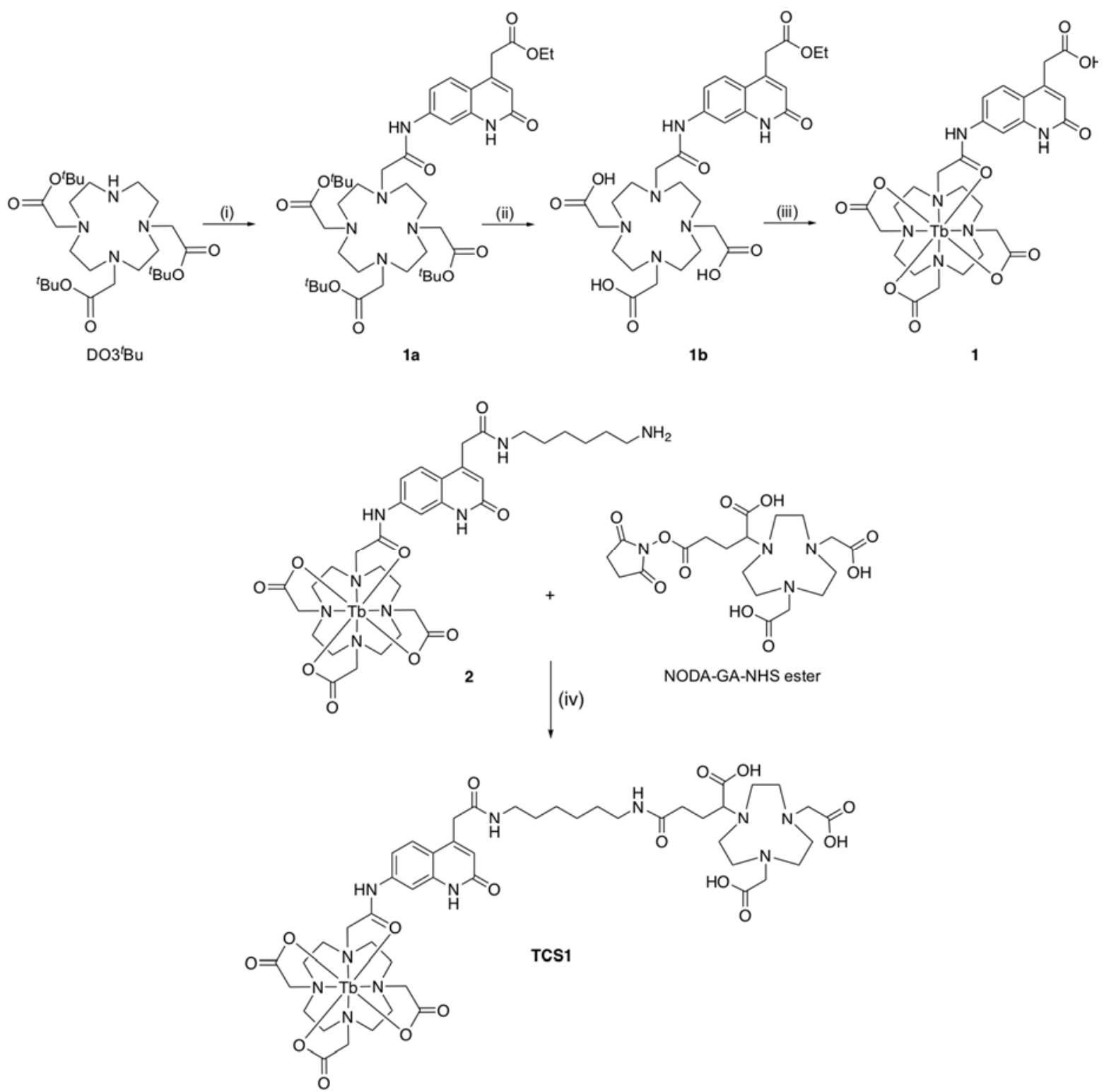

Scheme S1. Synthetic route for the preparation of the copper sensor TCS1. Reaction conditions: (i) 7-(2-Bromoacetylamino)-4-(carboethoxymethyl)quinolin-2(1H)-one, $\mathrm{K}_{2} \mathrm{CO}_{3}$, $\mathrm{KI}, \mathrm{CH}_{3} \mathrm{CN}, 80^{\circ} \mathrm{C}, 19$ h, $55 \%$; (ii) TFA, r.t., 1h, 80\%; (iii) a. $\mathrm{Tb}(\mathrm{OTf}) 3, \mathrm{H}_{2} \mathrm{O}$, r.t., 5 h, b. $\mathrm{NaOH}$, r.t., 2h, 43\%; (iv) Et 3 N, DMF, r.t., 20 h, $43 \%$. 


\section{$1.1 \quad$ Tb-complex 1}

This compound was synthesized according to literature procedures. ${ }^{1,2}$

\section{7-Amino-4-(carboethoxymethyl)quinolin-2(1H)-one}<smiles>CCOC(=O)Cc1cc(=O)[nH]c2cc(N)ccc12</smiles>

1,3-Phenylenediamine (5.00 g, $46.2 \mathrm{mmol})$ was dissolved in DMSO (35 mL) and diethyl-1,3acetonedicarboxylate $(9.18 \mathrm{~g}, 8.20 \mathrm{~mL}, 45.5 \mathrm{mmol})$ and $\mathrm{ZnCl}_{2}(6.37 \mathrm{~g}, 45.5 \mathrm{mmol})$ were added. The resulting mixture was stirred at $100{ }^{\circ} \mathrm{C}$ for $20 \mathrm{~h}$, when reaction control by TLC showed complete consumption of the starting material. The reaction mixture was diluted with $\mathrm{EtOH}$ $(35 \mathrm{~mL})$ and poured into an ice-cold $0.1 \mathrm{M}$ aq. solution of citric acid $(455 \mathrm{~mL})$. The formed precipitate was collected by vacuum filtration and washed with $\mathrm{H}_{2} \mathrm{O}(5 \times 20 \mathrm{~mL})$ and hot $\mathrm{CH}_{3} \mathrm{CN}(2 \times 25 \mathrm{~mL})$ to afford the product as a brown solid $(7.09 \mathrm{~g}, 28.8 \mathrm{mmol}, 63 \%)$.

TLC (EtOAc): $\mathrm{R}_{f}=0.34$.

Analytical UPLC (C18, $275 \mathrm{~nm}): \mathrm{R}_{t}=1.37 \mathrm{~min}$.

UPLC-MS $(\mathrm{C} 18,275 \mathrm{~nm}): \mathrm{R}_{t}(\mathrm{~m} / \mathrm{z})=0.96 \mathrm{~min}\left(247.1[\mathrm{M}+\mathrm{H}]^{+}, 493.2[2 \mathrm{M}+\mathrm{H}]^{+}\right)$.

${ }^{1}$ H NMR (d N $^{6}$ DMSO, $\left.400 \mathrm{MHz}\right) \delta: 11.27$ (s, $\left.1 \mathrm{H}, \mathrm{NH}\right), 7.25$ (d, J=8.4 Hz, $\left.1 A r-\mathrm{H}\right), 6.43$ (dd, $J=8.6 \mathrm{~Hz}, 2.6 \mathrm{~Hz}, 1 A r-\mathrm{H}), 6.37$ (d, $J=2.4 \mathrm{~Hz}, 1 A r-\mathrm{H}), 6.01$ (s, $1 \mathrm{H}, \mathrm{OCCH}), 5.79$ (s, $2 \mathrm{H}$, $\mathrm{NH}_{2}$ ), 4.09 (q, $\left.J=7.2 \mathrm{~Hz}, 2 \mathrm{H}, \mathrm{CH}_{2} \mathrm{CH}_{3}\right), 3.76$ (s, $\left.2 \mathrm{H}, \mathrm{CH}_{2} \mathrm{COO}\right), 1.17$ (t, $J=7.0 \mathrm{~Hz}, 3 \mathrm{H}$, $\left.\mathrm{CH}_{3}\right)$.

${ }^{13}$ C NMR (d ${ }^{6}$-DMSO, $\left.100.6 \mathrm{MHz}\right) \delta: 170.12\left(\mathrm{CH}_{2} \mathrm{C}=\mathrm{O}\right), 162.25(\mathrm{NC}=\mathrm{O}), 151.18(\operatorname{Ar}-\mathrm{C})$, 144.49 (Ar-C), 141.06 (Ar-C), 125.48 (Ar-C), 116.18 (Ar-C), 110.66 (Ar-C), 109.63 (Ar-C), 96.84 ( $\mathrm{Ar}-\mathrm{C}), 60.56\left(\mathrm{CH}_{2} \mathrm{CH}_{3}\right), 37.55\left(\mathrm{CH}_{2} \mathrm{CO}\right), 14.05\left(\mathrm{CH}_{3}\right)$.

\section{7-(2-Bromoacetylamino)-4-(carboethoxymethyl)quinolin-2(1H)-one}<smiles>CCOC(=O)Cc1cc(=O)[nH]c2cc(NC(=O)CBr)ccc12</smiles>

7-Amino-4-(carboethoxymethyl)quinolin-2(1H)-one $(3.15 \mathrm{~g}, 12.2 \mathrm{mmol})$ was dissolved in acetone $(300 \mathrm{~mL})$ and to this solution was added $\mathrm{NaHCO}_{3}(5.12 \mathrm{~g}, 61.0 \mathrm{mmol})$ and bromoacetyl bromide (4.92 g, $2.12 \mathrm{~mL}, 24.4 \mathrm{mmol})$. The reaction was stirred for $17 \mathrm{~h}$ at r.t. until reaction control by TLC showed complete consumption of the starting material. The 
solvent was then concentrated in vacuo to $c a .100 \mathrm{~mL}$, diluted with $\mathrm{H}_{2} \mathrm{O}(500 \mathrm{~mL})$ and filtered to collect the product as a brown solid (3.15 g, $8.57 \mathrm{mmol}, 70 \%)$.

TLC (EtOAc): $\mathrm{R}_{f}=0.5$.

Analytical UPLC (C18, $275 \mathrm{~nm}): \mathrm{R}_{t}=1.48 \mathrm{~min}$.

UPLC-MS (C18, $275 \mathrm{~nm}): \mathrm{R}_{t}(\mathrm{~m} / \mathrm{z})=1.06 \mathrm{~min}\left(367.2[\mathrm{M}+\mathrm{H}]^{+}, 735.2[2 \mathrm{M}+\mathrm{H}]^{+}\right)$.

${ }^{1}$ H NMR (d $\mathrm{d}^{6}$-DMSO, $\left.400 \mathrm{MHz}\right) \delta: 11.74$ (s, $\left.1 \mathrm{H}, \mathrm{NH}\right), 10.66$ (s, $\left.1 \mathrm{H}, \mathrm{NH}\right), 7.76$ (d, J=2.0 Hz, $1 A r-\mathrm{H}), 7.57(\mathrm{~d}, J=8.2 \mathrm{~Hz}, 1 A r-\mathrm{H}), 7.30(\mathrm{dd}, J=8.8 \mathrm{~Hz}, 2.0 \mathrm{~Hz}, 1 A r-\mathrm{H}), 6.37(\mathrm{~s}, 1 \mathrm{H}$, $\mathrm{OCCH}$ ), 4.10 (q, J=7.2 Hz, $\left.2 \mathrm{H}, \mathrm{CH}_{2} \mathrm{CH}_{3}\right), 4.07$ (s, $2 \mathrm{H}, \mathrm{CH}_{2} \mathrm{Br}$ ), 3.91 (s, $2 \mathrm{H}, \mathrm{CH}_{2} \mathrm{COO}$ ), 1.17 (t, $\left.J=7.1 \mathrm{~Hz}, 3 \mathrm{H}, \mathrm{CH}_{3}\right)$.

${ }^{13}$ C NMR (d ${ }^{6}$-DMSO, 100.6 MHz) $\delta: 169.87(C=\mathrm{O}), 165.24(\mathrm{C}=\mathrm{O}), 161.81(\mathrm{NC}=\mathrm{O}), 144.21$ (Ar-C), 140.33 (Ar-C), 139.68 (Ar-C), 125.46 (Ar-C), 121.17 (Ar-C), 115.23 (Ar-C), 113.65 (Ar-C), 104.85 ( $A r-\mathrm{C}), 60.68\left(\mathrm{CH}_{2} \mathrm{CH}_{3}\right), 37.30\left(\mathrm{CH}_{2} \mathrm{CO}\right), 30.34\left(\mathrm{CH}_{2} \mathrm{Br}\right), 14.02\left(\mathrm{CH}_{3}\right)$.

\section{1,4,7-Tris(tert-butoxycarbonylmethyl)-10-((7'-acetamide)-4'-(carboethoxymethyl) quinolin-2'(1H)-one)-1,4,7,10-tetraazacyclododecane (1a)}

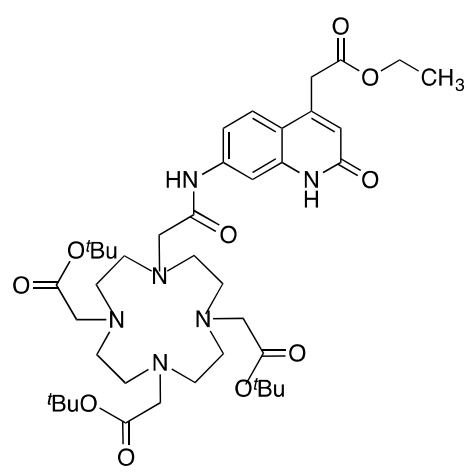

To a solution of $\mathrm{DO}^{t} \mathrm{Bu}(196 \mathrm{mg}, 0.381 \mathrm{mmol})$ in $\mathrm{CH}_{3} \mathrm{CN}(20 \mathrm{~mL})$ was added $\mathrm{K}_{2} \mathrm{CO}_{3}(270$ $\mathrm{mg}, \quad 1.95 \mathrm{mmol})$, KI (128 $\mathrm{mg}, \quad 0.771 \mathrm{mmol})$ and 7-(2-Bromoacetylamino)-4(carboethoxymethyl)quinolin-2(1H)-one $(140 \mathrm{mg}, 0.381 \mathrm{mmol})$. The resulting mixture was stirred for $2 \mathrm{~h}$ at r.t. and then heated to $80{ }^{\circ} \mathrm{C}$ for $19 \mathrm{~h}$, when reaction control by analytical UPLC showed complete consumption of the carbostyril educt. The reaction mixture was filtered and the solvent of the filtrate was evaporated in vacuo. After purification by silica gel chromatography using DCM with an increasing gradient of $\mathrm{MeOH}(0-10 \%)$, 1a was obtained as a yellow solid (170 $\mathrm{mg}, 0.212 \mathrm{mmol}, 55 \%)$.

Analytical UPLC $(\mathrm{C} 18,275 \mathrm{~nm}): \mathrm{R}_{t}=1.50 \mathrm{~min}$.

UPLC-MS $(\mathrm{C} 18,275 \mathrm{~nm}): \mathrm{R}_{t}(\mathrm{~m} / \mathrm{z})=1.04 \min \left(801.6[\mathrm{M}+\mathrm{H}]^{+}, 401.3[\mathrm{M}+2 \mathrm{H}]^{2+}\right)$. 
${ }^{1} \mathbf{H}$ NMR $\left(\mathrm{CDCl}_{3}, 400 \mathrm{MHz}\right) \delta: 11.28(\mathrm{~s}, 1 \mathrm{H}, \mathrm{NH}), 7.84-7.83(\mathrm{~m}, 1 \mathrm{Ar}-\mathrm{H}), 7.65$ (br s, 1 ArH), 7.44-7.41 (m, $1 A r-\mathrm{H}), 6.51$ (s, $1 \mathrm{H}, \mathrm{OCCH}), 4.46-2.97$ (br m, $\left.26 \mathrm{H}, 13 \times \mathrm{CH}_{2}\right), 4.15$ (q, $J$ $\left.=7.1 \mathrm{~Hz}, 2 \mathrm{H}, \mathrm{CH}_{2} \mathrm{CH}_{3}\right), 1.49-1.29$ (br m, $\left.27 \mathrm{H}, \mathrm{C}\left(\mathrm{CH}_{3}\right)_{3}\right), 1.23$ (t, J=7.1 Hz, $\left.3 \mathrm{H}, \mathrm{CH}_{2} \mathrm{CH}_{3}\right)$. ${ }^{13} \mathbf{C ~ N M R}\left(\mathrm{CDCl}_{3}, 100.6 \mathrm{MHz}\right) \delta: 172.68(\mathrm{C}=\mathrm{O}), 172.35(\mathrm{C}=\mathrm{O}), 171.43(\mathrm{C}=\mathrm{O}), 170.45(\mathrm{C}=\mathrm{O})$, $169.48(\mathrm{C}=\mathrm{O}), 162.70(\mathrm{NC}=\mathrm{O}), 144.48(A r-\mathrm{C}), 141.28$ (Ar-C), $138.76(A r-\mathrm{C}), 124.34(A r-\mathrm{C})$, 120.43 (Ar-C), 115.54 (Ar-C), 115.43 (Ar-C), 105.71 (Ar-C), $81.77\left(C\left(\mathrm{CH}_{3}\right)_{3}\right), 81.43$ $\left(C\left(\mathrm{CH}_{3}\right)_{3}\right), 61.15\left(\mathrm{CH}_{2}\right), 57.68\left(\mathrm{CH}_{2}\right), 56.91\left(\mathrm{CH}_{2}\right), 55.57\left(\mathrm{CH}_{2}\right), 55.45\left(\mathrm{CH}_{2}\right), 52.40\left(\mathrm{br}, \mathrm{CH}_{2}\right)$, $51.60\left(\mathrm{CH}_{2}\right), 51.07\left(\mathrm{CH}_{2}\right), 50.04\left(\mathrm{CH}_{2}\right), 49.52\left(\mathrm{CH}_{2}\right), 49.01\left(\mathrm{CH}_{2}\right), 47.07\left(\mathrm{CH}_{2}\right), 46.37\left(\mathrm{CH}_{2}\right)$, $\left.\left.38.59\left(\mathrm{CH}_{2} \mathrm{CO}\right), 28.03\left(\mathrm{C}\left(\mathrm{CH}_{3}\right)_{3}\right), 27.79\left(\mathrm{C}_{(\mathrm{CH}}\right)_{3}\right), 27.27\left(\mathrm{C} \mathrm{CH}_{3}\right)_{3}\right), 13.95\left(\mathrm{CH}_{2} \mathrm{CH}_{3}\right)$.

\section{1,4,7-Tris(carbonylmethyl)-10-((7'-acetamide)-4'-(carboethoxymethyl)quinolin-2'(1H)- one)-1,4,7,10-tetraazacyclododecane (1b)}

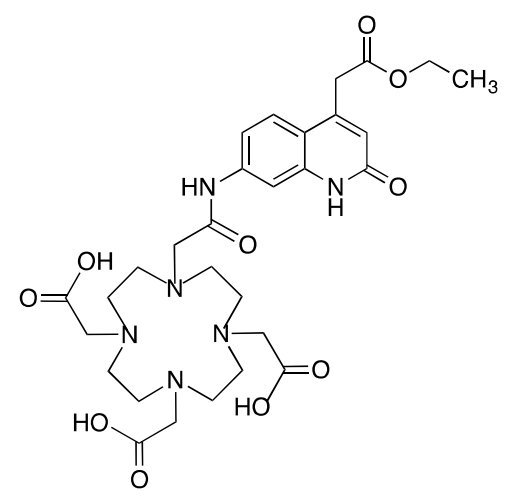

Compound 1a (325 mg, $0.405 \mathrm{mmol})$ was dissolved in TFA (15 mL) and the resulting mixture was stirred for $1 \mathrm{~h}$ at r.t. After evaporation via a gentle stream of $\mathrm{N}_{2}, \mathrm{H}_{2} \mathrm{O}(10 \mathrm{~mL})$ was added and the obtained suspension was neutralized with $a q$. $\mathrm{NaOH}(5 \mathrm{M})$. This mixture was purified directly using reversed-phase silica gel chromatography with a gradient of $5-100 \% \mathrm{CH}_{3} \mathrm{CN}$ in $\mathrm{H}_{2} \mathrm{O} / \mathrm{TFA}(0.1 \%)$. Lyophilization of the combined fractions afforded the $\mathbf{1 b}$ as a yellowish powder (205 mg, $0.324 \mathrm{mmol}, 80 \%)$.

Analytical UPLC (C18, $275 \mathrm{~nm}): \mathrm{R}_{t}=1.31 \mathrm{~min}$.

UPLC-MS $(\mathrm{C} 18,275 \mathrm{~nm}): \mathrm{R}_{t}(\mathrm{~m} / \mathrm{z})=0.76 \min \left(633.3[\mathrm{M}+\mathrm{H}]^{+}\right)$.

IR (neat; 3500-600 $\mathrm{cm}^{-1}$ ): $\mathrm{v}_{\max }=3095 w, 2990 w, 1723 \mathrm{~m}, 1652 \mathrm{~s}, 1645 \mathrm{~s}, 1539 \mathrm{~m}, 1458 w, 1386 \mathrm{~m}$, $1183 s, 1129 s, 1087 m, 1021 w, 993 w, 874 w, 825 m, 798 m, 719 m, 680 m, 667 m, 619 w, 609 w$.

${ }^{1} \mathbf{H}$ NMR (d $\mathrm{d}^{6}$-DMSO, $\left.400 \mathrm{MHz}\right) \delta: 11.65$ (s, $\left.1 \mathrm{H}, \mathrm{NH}\right), 10.71$ (s, $\left.1 \mathrm{H}, \mathrm{NH}\right), 7.58-7.55(\mathrm{~m}, 2 \mathrm{H}$, $2 A r-\mathrm{H}), 7.46$ (d-like m, “ $J$ ' = 8.7 Hz, $1 A r-\mathrm{H}), 6.38(\mathrm{~s}, 1 \mathrm{H}, \mathrm{OCCH}), 4.10(\mathrm{q}, J=7.1 \mathrm{~Hz}, 2 \mathrm{H}$, $\mathrm{CH}_{2} \mathrm{CH}_{3}$ ), 3.97-3.21 (br m, $26 \mathrm{H}, 13 \times \mathrm{CH}_{2}$, overlapping with $\mathrm{H}_{2} \mathrm{O}$ peak), 1.17 (t, $J=7.1 \mathrm{~Hz}$, $\left.3 \mathrm{H}_{1} \mathrm{CH}_{3}\right)$. 
${ }^{13}$ C NMR (d -DMSO, 100.6 MHz) $\delta: 169.92(\mathrm{C}=\mathrm{O}), 161.86(\mathrm{C}=\mathrm{O}), 144.29(\operatorname{Ar}-\mathrm{C}), 140.30$ (Ar-C), 139.54 (Ar-C), 125.53 (Ar-C), 121.19 (Ar-C), 115.63 (Ar-C), $113.92(A r-C), 104.65$ (Ar-C), $60.73\left(\mathrm{CH}_{2}\right), 55.29\left(\mathrm{CH}_{2}\right), 54.14\left(\mathrm{CH}_{2}\right), 53.90\left(\mathrm{br}, \mathrm{CH}_{2}\right), 53.24$ (br, $\left.\mathrm{CH}_{2}\right), 51.83\left(\mathrm{CH}_{2}\right)$, 50.10 (br, $\left.\mathrm{CH}_{2}\right), 49.29$ (br, $\left.\mathrm{CH}_{2}\right), 37.33\left(\mathrm{CH}_{2} \mathrm{CO}\right), 14.04\left(\mathrm{CH}_{2} \mathrm{CH}_{3}\right)$.

\section{Tb-Complex 1}

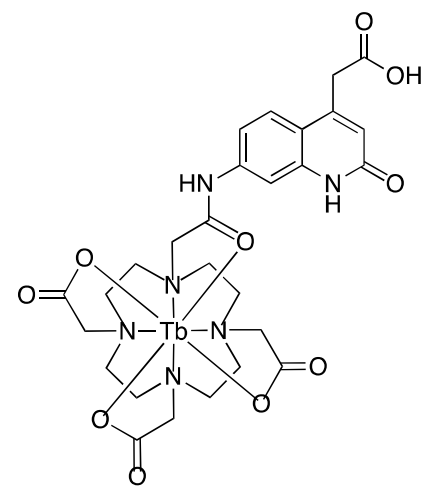

Compound $\mathbf{1 b}$ (56 mg, $0.070 \mathrm{mmol})$ was dissolved in $\mathrm{H}_{2} \mathrm{O}(10 \mathrm{~mL})$ and to this solution was added $\mathrm{Tb}(\mathrm{OTf})_{3}(42 \mathrm{mg}, 0.070 \mathrm{mmol})$. The $\mathrm{pH}$ of the mixture was adjusted to 6 using aq. $\mathrm{NaOH}$ (1 M) and the resulting solution was stirred for $5 \mathrm{~h}$ at r.t. until full complexation was observed by analytical UPLC. The solution was then basified to $\mathrm{pH} 12$ using aq. $\mathrm{NaOH}(5 \mathrm{M})$ and stirred for a further $2 \mathrm{~h}$ when full cleavage of the ester was observed by analytical UPLC. The $\mathrm{pH}$ was then adjusted to 7 using aq. $\mathrm{HCl}(2 \mathrm{M})$ and directly purified using reversed-phase silica gel chromatography with a gradient of $5-100 \% \mathrm{CH}_{3} \mathrm{CN}$ in $\mathrm{H}_{2} \mathrm{O} / \mathrm{TFA}(0.1 \%)$. Lyophilization of the combined fractions afforded 1 as a white powder (23 $\mathrm{mg}, 0.030 \mathrm{mmol}, 43 \%)$.

Analytical UPLC (C18, $275 \mathrm{~nm}): \mathrm{R}_{t}=1.20 \mathrm{~min}$.

UPLC-MS $(\mathrm{C} 18,275 \mathrm{~nm}): \mathrm{R}_{t}(\mathrm{~m} / \mathrm{z})=0.70 \min \left(761.3[\mathrm{M}+\mathrm{H}]^{+}, 381.1[\mathrm{M}+2 \mathrm{H}]^{2+}\right)$.

HR-MS $(m / z)$ : calcd for $\mathrm{C}_{27} \mathrm{H}_{34} \mathrm{~N}_{6} \mathrm{O}_{10} \mathrm{~Tb}[\mathrm{M}+\mathrm{H}]^{+}$761.15844, found: 761.15816.

IR (neat; 4000-600 $\mathrm{cm}^{-1}$ ): $V_{\max }=2879 w, 1588 s, 1462 w, 1405 w, 1318 w, 1179 m, 1083 \mathrm{~m}$, $1002 w, 971 w, 935 w, 823 m, 677 s, 618 m$.

Elemental analysis calc. for $\mathrm{C}_{2} 7 \mathrm{H}_{33} \mathrm{~N}_{6} \mathrm{O}_{8} \mathrm{~Tb}\left(\mathrm{CF}_{3} \mathrm{COOH}\right)\left(\mathrm{H}_{2} \mathrm{O}\right)_{2}(\%)$ : $\mathrm{C}, 38.25 ; \mathrm{H}, 4.21 ; \mathrm{N}$, 9.23; found: C, 37.95; H, 4.60; N, 9.36. 


\subsection{Copper sensor TCS1}

\section{1,4,7-Tris(carbonylmethyl)-10-((7'-acetamide)-4'-(aminohexylamidomethyl)quinolin-}

2'(1H)-one)-1,4,7,10-tetraazacyclododecane terbium(III) (2)

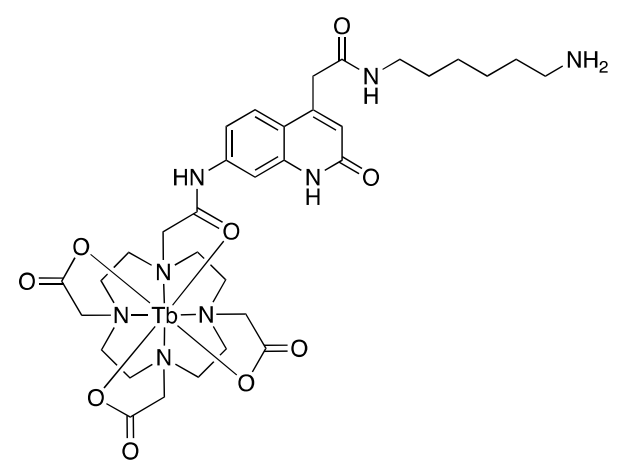

Complex 1 (25 mg, $0.027 \mathrm{nmol}$ ) was dissolved in DMSO (5 mL) and to this mixture was added Et $3 \mathrm{~N}$ (11 mg, $15 \mu \mathrm{L}, 0.11 \mathrm{mmol})$, HATU (42 mg, $0.11 \mathrm{mmol}$ ) and 1,6-diaminohexane (6.5 mg, $0.055 \mathrm{mmol}$ ). The solution was stirred for $17 \mathrm{~h}$ at r.t. and then purified by preparative HPLC. The combined fractions were lyophilized to afford a white powder ( $22 \mathrm{mg}, 0.025 \mathrm{mmol}, 93 \%$ ). Analytical UPLC (C18, $275 \mathrm{~nm}): \mathrm{R}_{t}=1.22 \mathrm{~min}$.

UPLC-MS $(\mathrm{C} 18,275 \mathrm{~nm}): \mathrm{R}_{t}(\mathrm{~m} / \mathrm{z})=0.78 \mathrm{~min}\left(430.3[\mathrm{M}+2 \mathrm{H}]^{2+}, 859.3[\mathrm{M}+\mathrm{H}]^{+}\right)$.

HR-MS $(\mathrm{m} / \mathrm{z})$ : calc. for $\mathrm{C}_{33} \mathrm{H}_{48} \mathrm{~N}_{8} \mathrm{O}_{9} \mathrm{~Tb}[\mathrm{M}+\mathrm{H}]^{+}$859.27922, found: 859.27875.

IR (neat, $4000-600 \mathrm{~cm}^{-1}$ ): $\mathrm{V}_{\max }=3295 w, 3084 w, 2937 w, 2868 w, 1587 \mathrm{~s}, 1558 \mathrm{~m}, 1458 w, 1403 \mathrm{~m}$, $1319 w, 1199 s, 1178 s, 1128 s, 1083 s, 1002 w, 969 w, 939 w, 905 w, 885 w, 834 m, 799 m, 719 s$, $663 w$.

\section{TCS1}

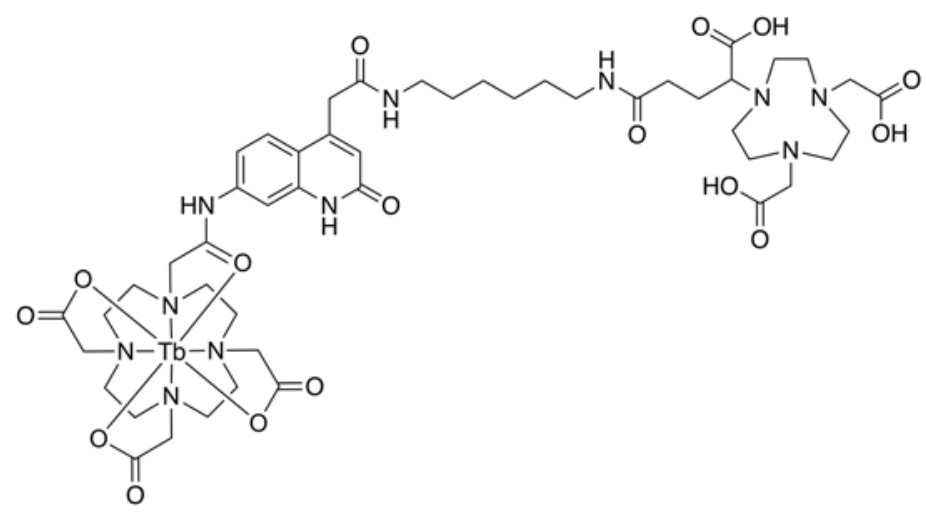

Complex 2 (20 mg, $0.023 \mathrm{mmol}$ ) was dissolved in DMF (5 mL) and to this mixture was added NODA-GA-NHS ester $(28 \mathrm{mg}, 0.038 \mathrm{mmol})$ and $\mathrm{Et}_{3} \mathrm{~N}(10 \mathrm{mg}, 14 \mu \mathrm{L}, 0.10 \mathrm{mmol})$. The solution was stirred for $20 \mathrm{~h}$ at r.t. and then purified by preparative HPLC. The combined fractions were lyophilized to afford a white powder (12 mg, $9.9 \mu \mathrm{mol}, 43 \%)$. 
Analytical UPLC (C18, $275 \mathrm{~nm}): \mathrm{R}_{t}=1.29 \mathrm{~min}$.

UPLC-MS $(\mathrm{C} 18,275 \mathrm{~nm}): \mathrm{R}_{t}(\mathrm{~m} / z)=0.77 \min \left(609.0[\mathrm{M}+2 \mathrm{H}]^{2+}, 1216.4[\mathrm{M}+\mathrm{H}]^{+}\right)$.

HR-MS $(\mathrm{m} / z)$ : calc. for $\mathrm{C}_{48} \mathrm{H}_{72} \mathrm{~N}_{11} \mathrm{O}_{16} \mathrm{~Tb}[\mathrm{M}+2 \mathrm{H}]^{2+}$ 608.72005, found: 608.72098 .

IR (neat, $3500-600 \mathrm{~cm}^{-1}$ ): $V_{\max }=2987 w, 2884 w, 1653 s, 1617 s, 1592 s, 1554 m, 1459 \mathrm{w}, 1404 w$, $1321 w, 1246 w, 1176 s, 1127 s, 1084 w, 969 w, 938 w, 886 w, 831 w, 798 m, 719 s, 680 m, 667 m$.

Elemental analysis calc. for $\mathrm{C}_{48} \mathrm{H}_{70} \mathrm{~N}_{11} \mathrm{O}_{16} \mathrm{~Tb}\left(\mathrm{CF}_{3} \mathrm{COOH}\right)_{1.5}\left(\mathrm{H}_{2} \mathrm{O}\right)_{2}(\%): \mathrm{C}, 43.04 ; \mathrm{H}, 5.35$; N, 10.83; found: C, 43.34; H, 5.74; N, 10.57.

TCS2

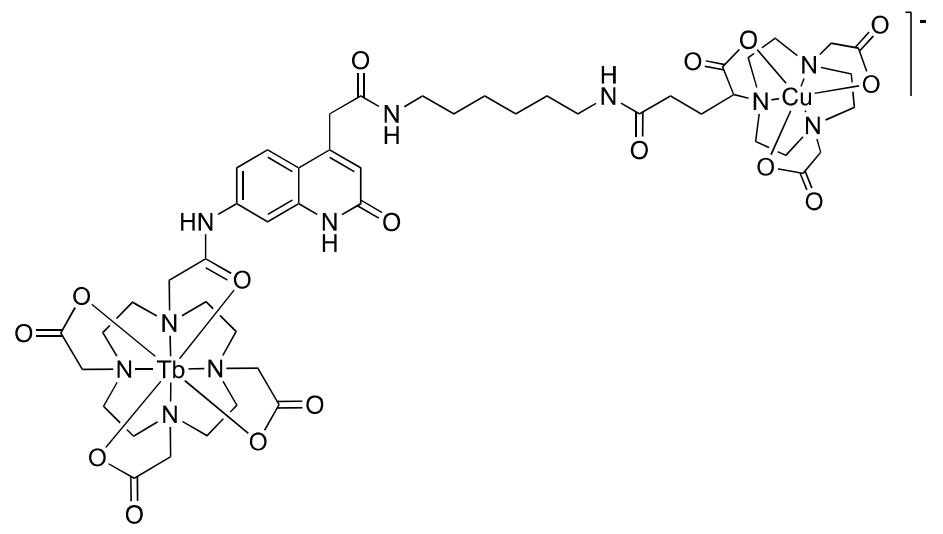

TCS2 was prepared in situ by addition of 1 equivalent of $\mathrm{CuCl}_{2}$ to TCS1 in $\mathrm{H}_{2} \mathrm{O}$ at r.t. The reaction can be followed by analytical UPLC where complete conversion was indicated within minutes after the addition of $\mathrm{CuCl}_{2}$.

Analytical UPLC $(\mathrm{C} 18,275 \mathrm{~nm}): \mathrm{R}_{t}=1.26 \mathrm{~min}$.

UPLC-MS $(\mathrm{C} 18,275 \mathrm{~nm}): \mathrm{R}_{t}(\mathrm{~m} / \mathrm{z})=0.74 \min \left(639.7[\mathrm{M}+3 \mathrm{H}]^{2+}, 1277.4[\mathrm{M}+2 \mathrm{H}]^{+}\right)$.

HR-MS $(m / z)$ : calc. for $\mathrm{C}_{48} \mathrm{H}_{70} \mathrm{CuN}_{11} \mathrm{O}_{16} \mathrm{~Tb}[\mathrm{M}+3 \mathrm{H}]^{2+}$ 639.17702, found: 639.17701. 


\section{Spectra}

\subsection{NMR Spectroscopy}
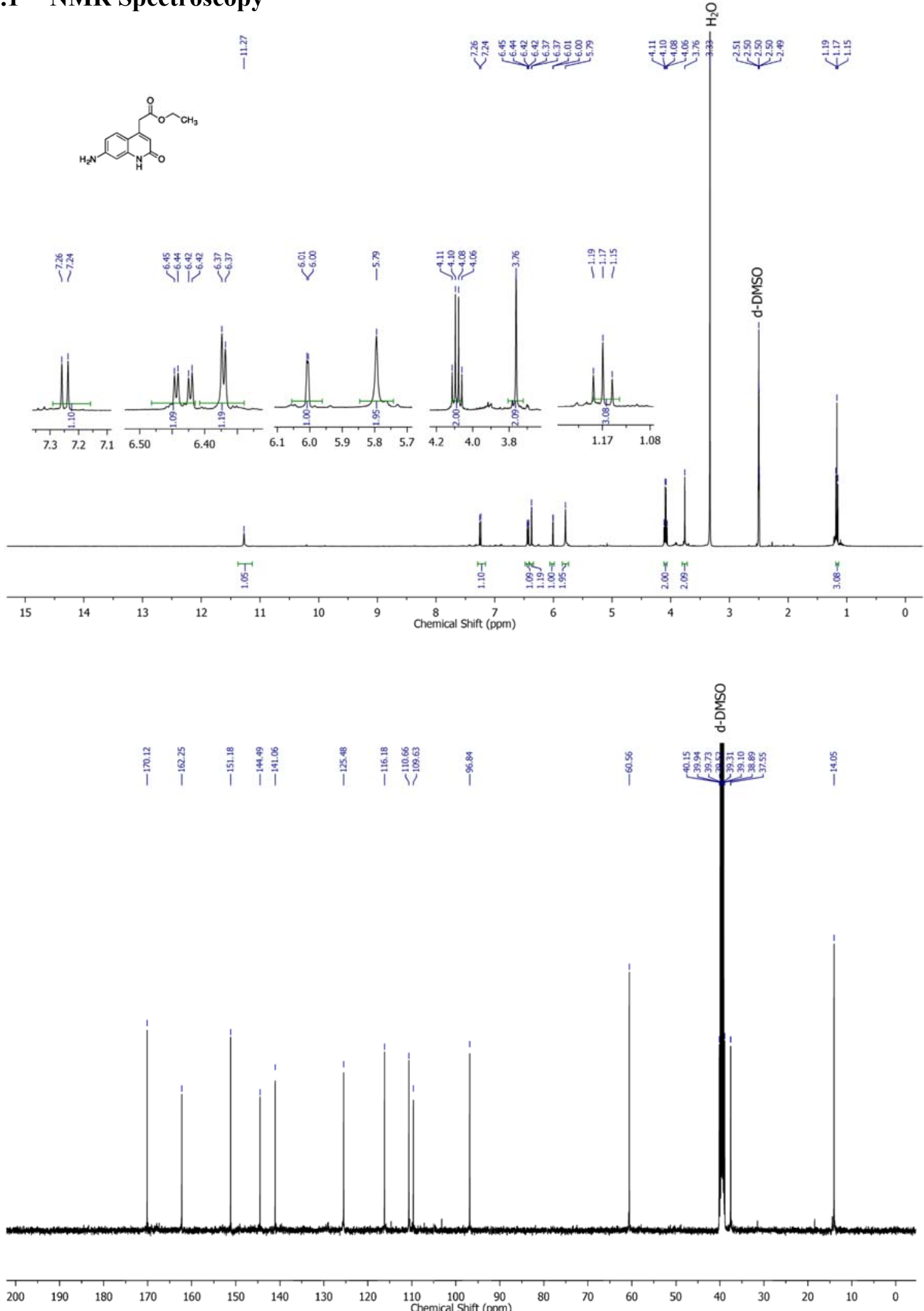

Figure S1. ${ }^{1} \mathrm{H}$ and ${ }^{13} \mathrm{C}$ NMR ( $\mathrm{d}_{6}$-DMSO) of 7-Amino-4-(carboethoxymethyl)quinolin-2(1H)-one. 

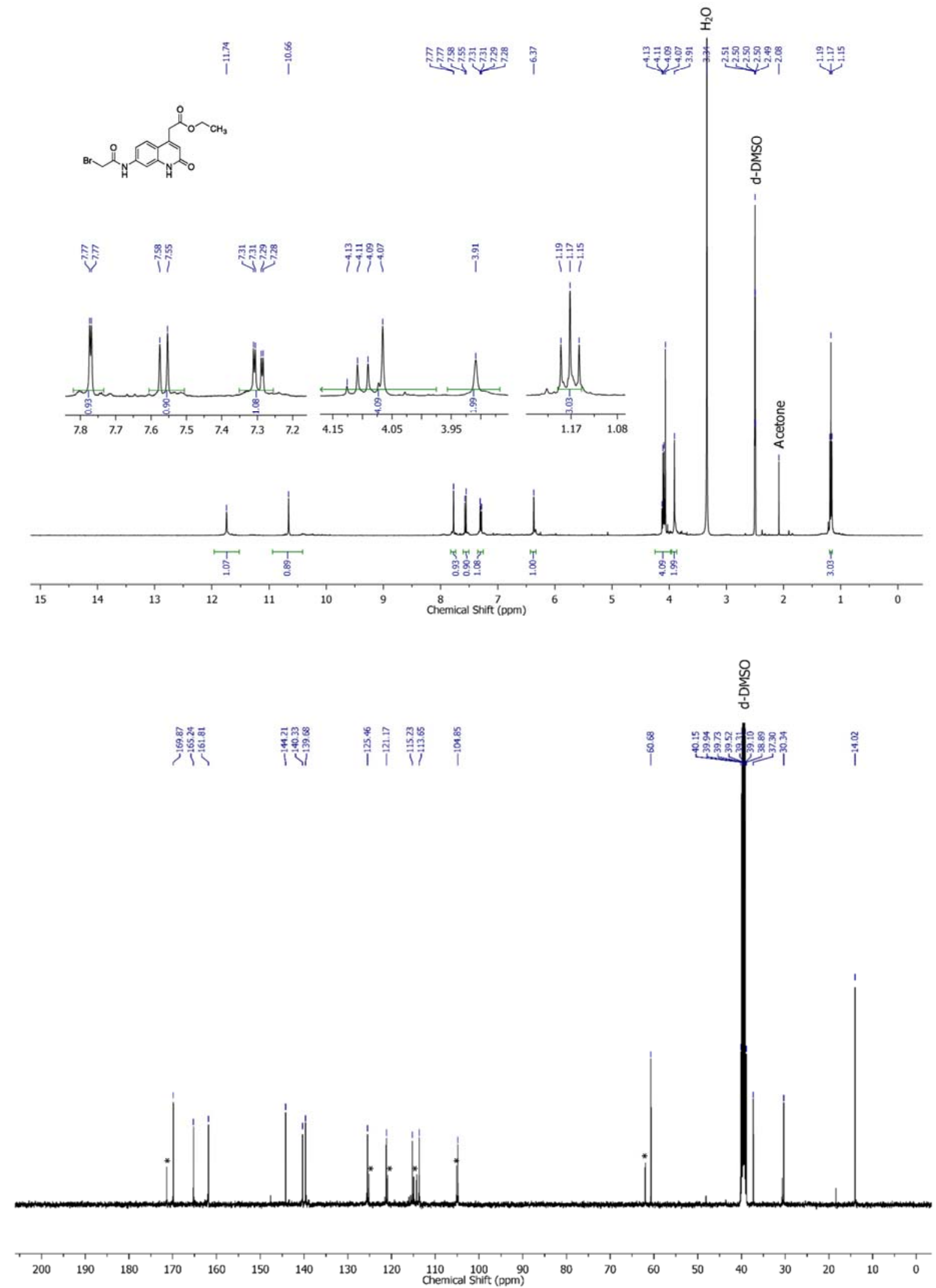

Figure S2. ${ }^{1} \mathrm{H}$ and ${ }^{13} \mathrm{C}$ NMR (d6-DMSO) of 7-(2-Bromoacetylamino)-4-(carboethoxymethyl)quinolin$2(1 \mathrm{H})$-one (signals for hydrolysis product marked with asterisks in $\left.{ }^{13} \mathrm{C} N M R\right)$. 

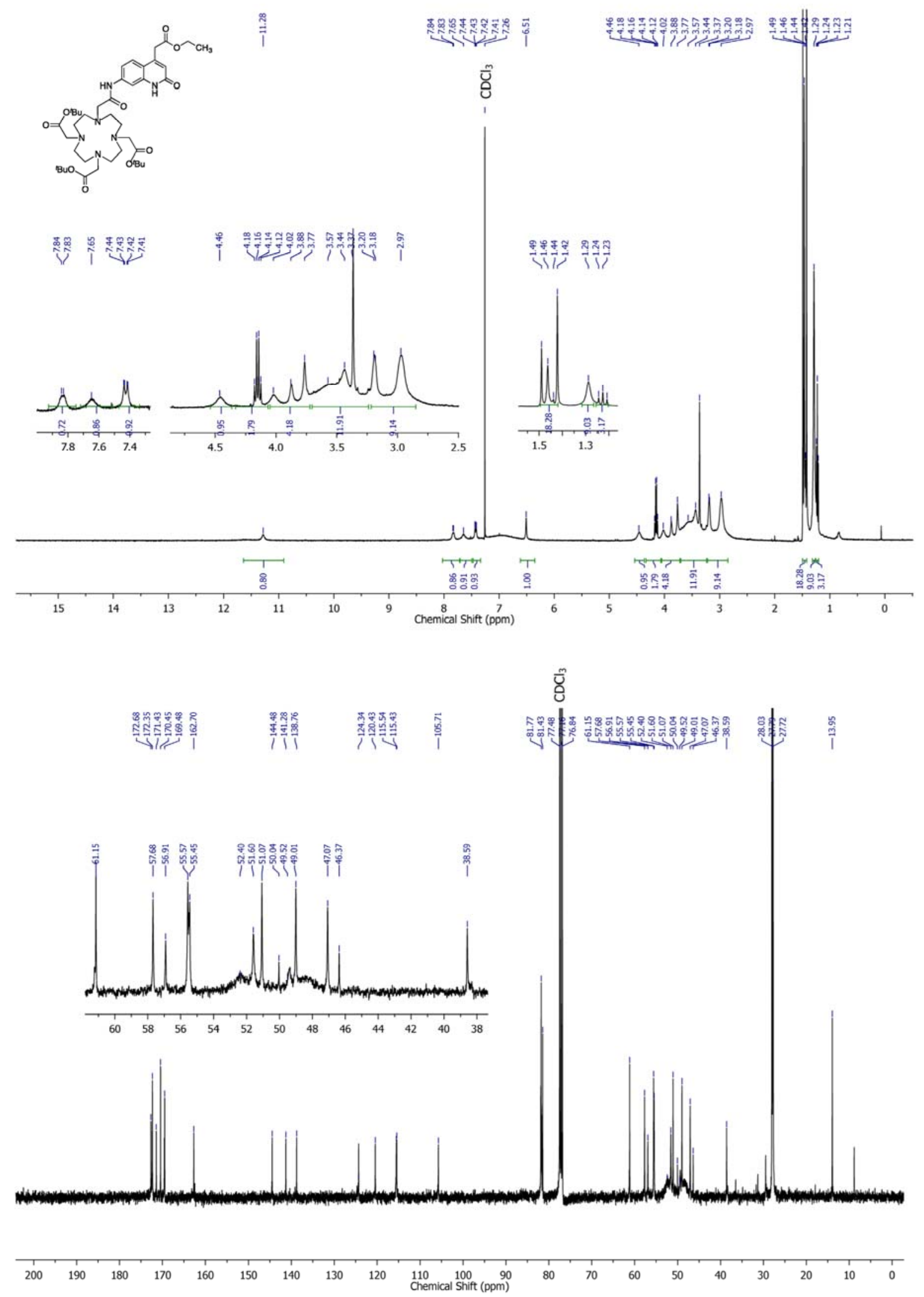

Figure S3. ${ }^{1} \mathrm{H}$ and ${ }^{13} \mathrm{C} \mathrm{NMR}\left(\mathrm{CDCl}_{3}\right)$ of $\mathbf{1 a}$. 

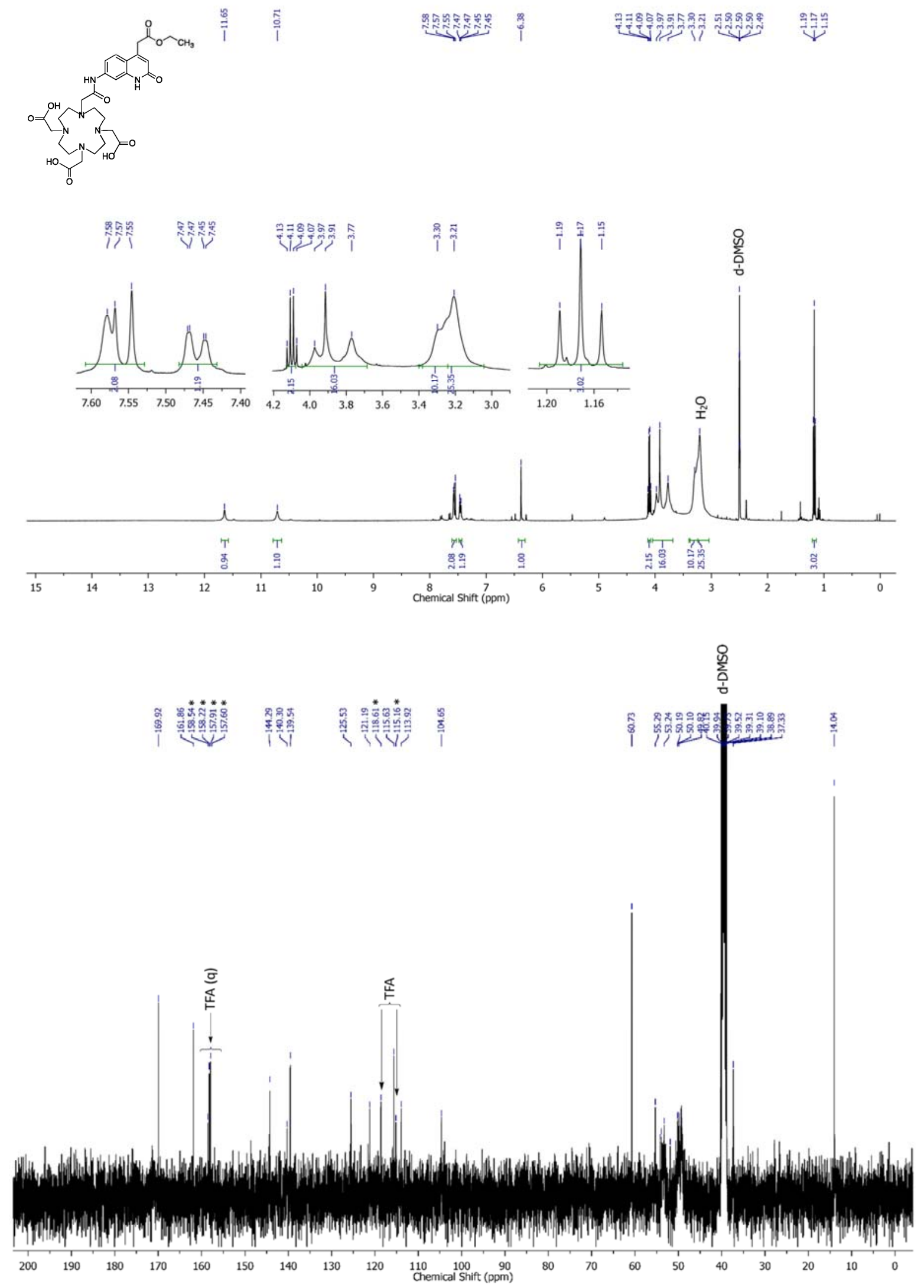

Figure S4. ${ }^{1} \mathrm{H}$ and ${ }^{13} \mathrm{C}$ NMR ( $\mathrm{d}_{6}$-DMSO) of $\mathbf{1 b}$, obtained as TFA salt. TFA signals in ${ }^{13} \mathrm{C}$ NMR marked with asterisks. 


\subsection{Mass Spectrometry}
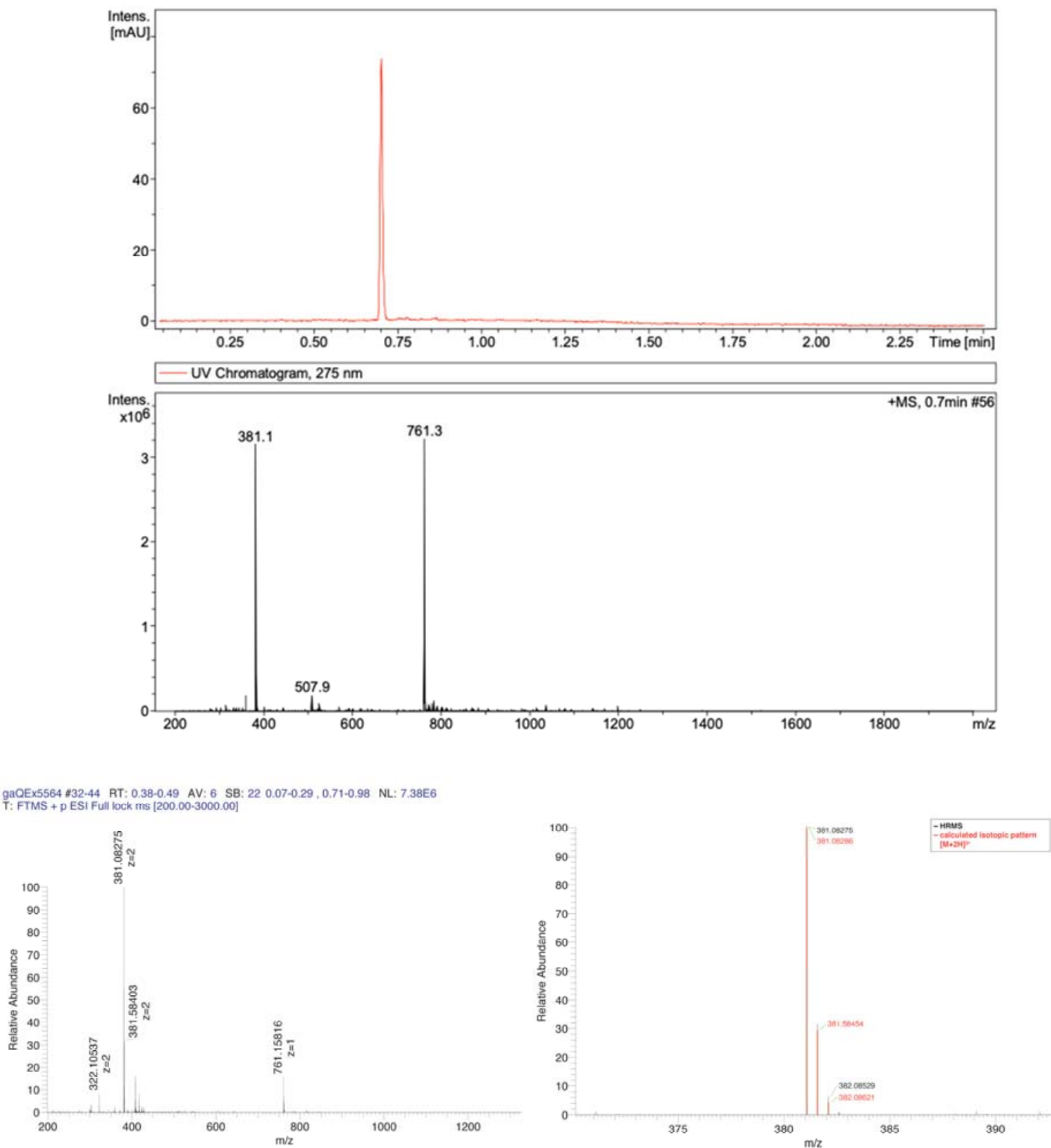

Figure S5. UPLC-MS and HR-MS of 1. 

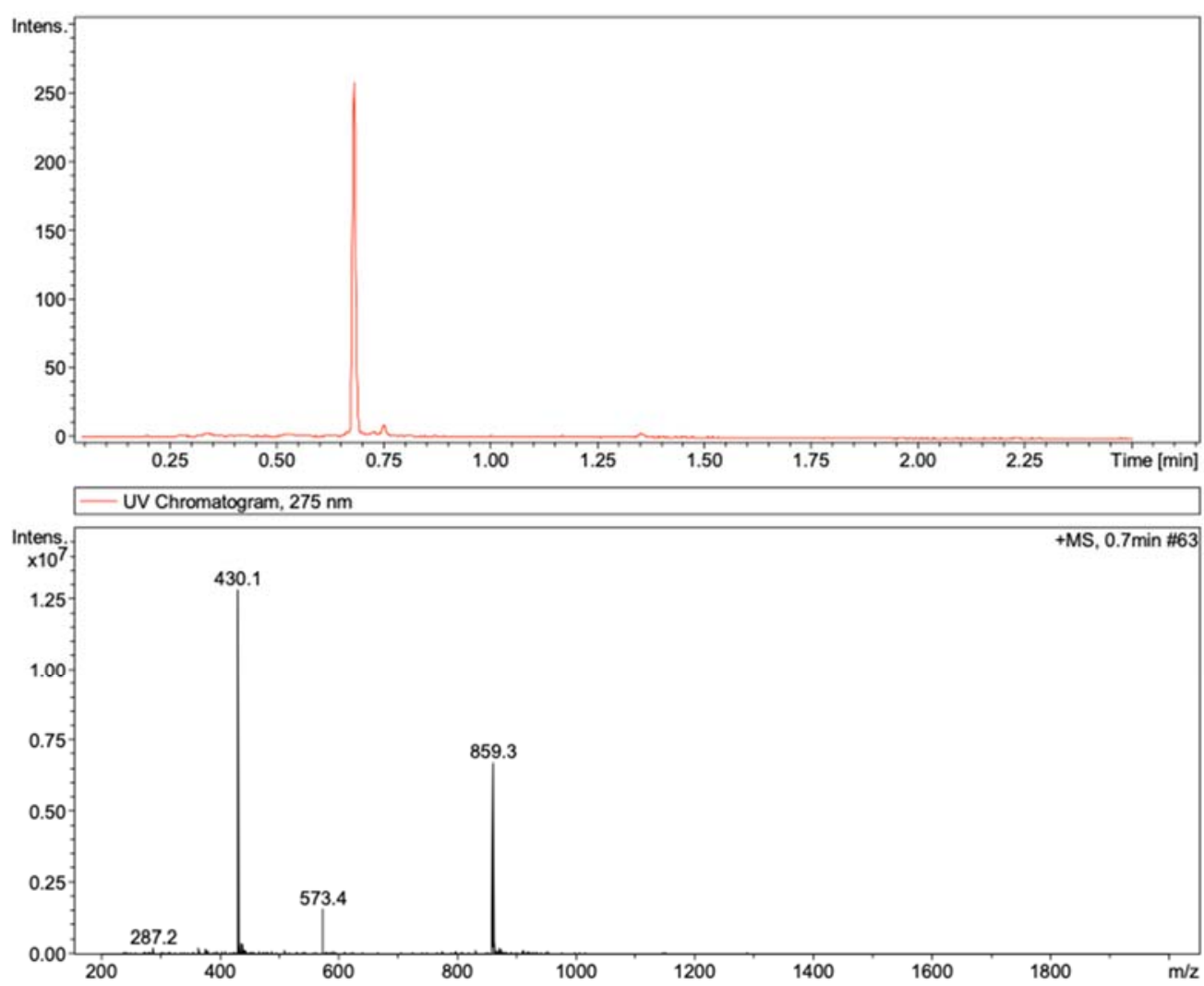

gaOEx5414 \#34-48 RT: 0.38-0.49 AV: 7 SB: 23 0.07-0.29, 0.71-0.98 NL: 4.21E7
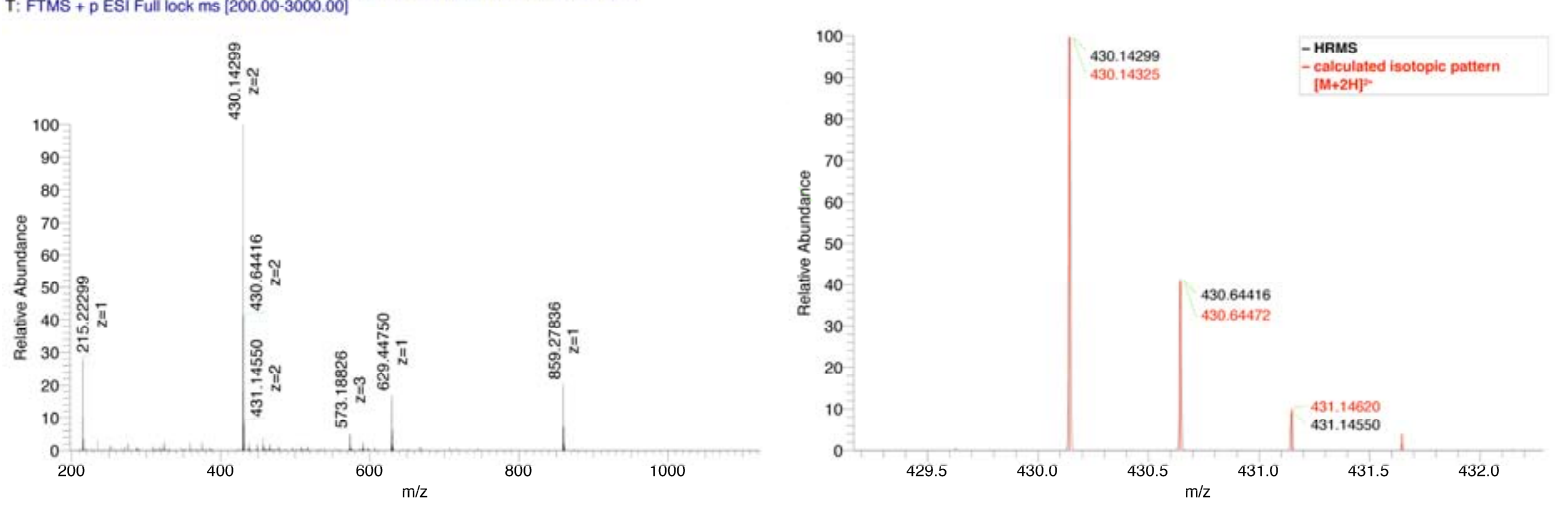

Figure S6. UPLC-MS and HR-MS of 2. 

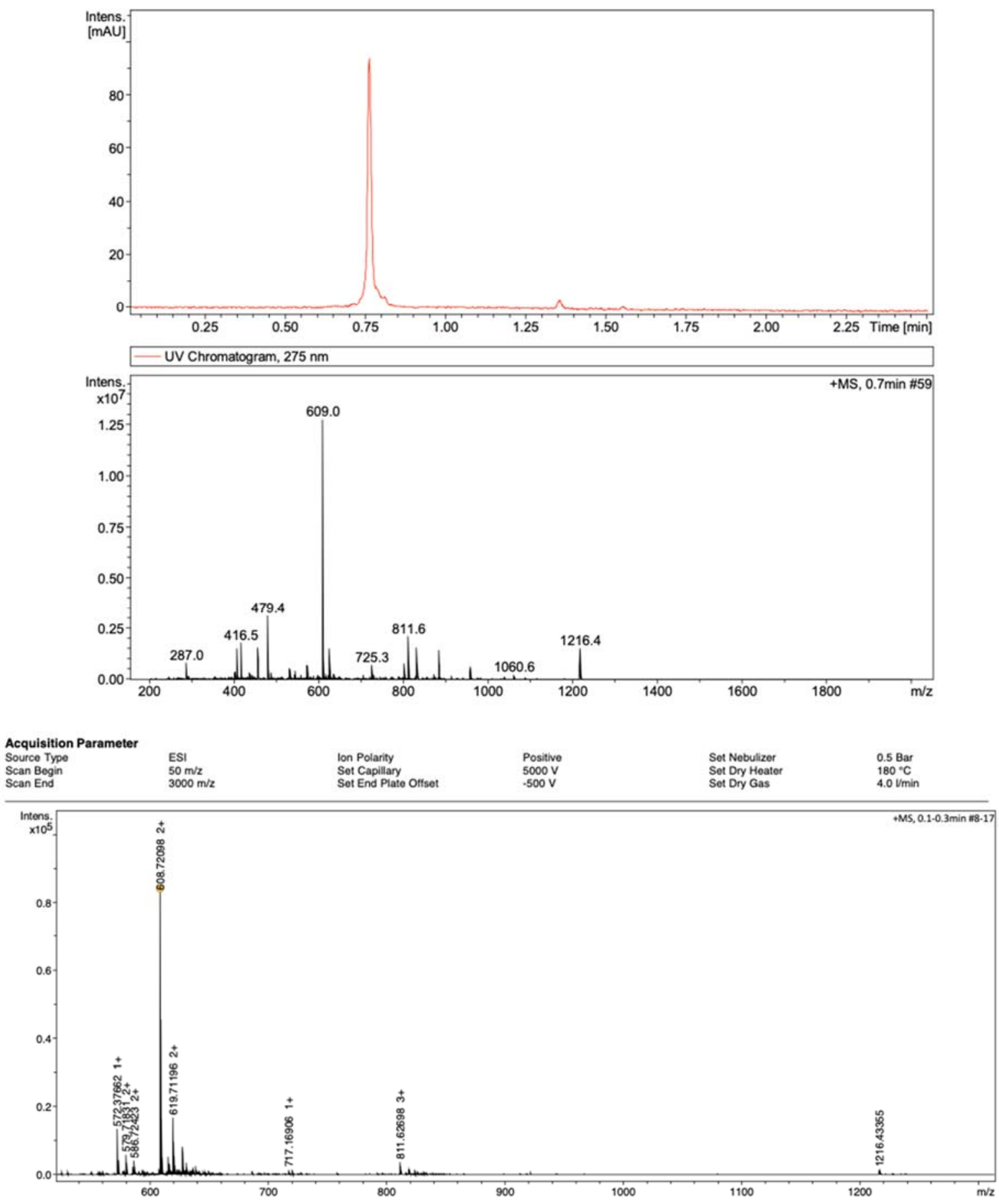

Figure S7. UPLC-MS and HR-MS of TCS1. 

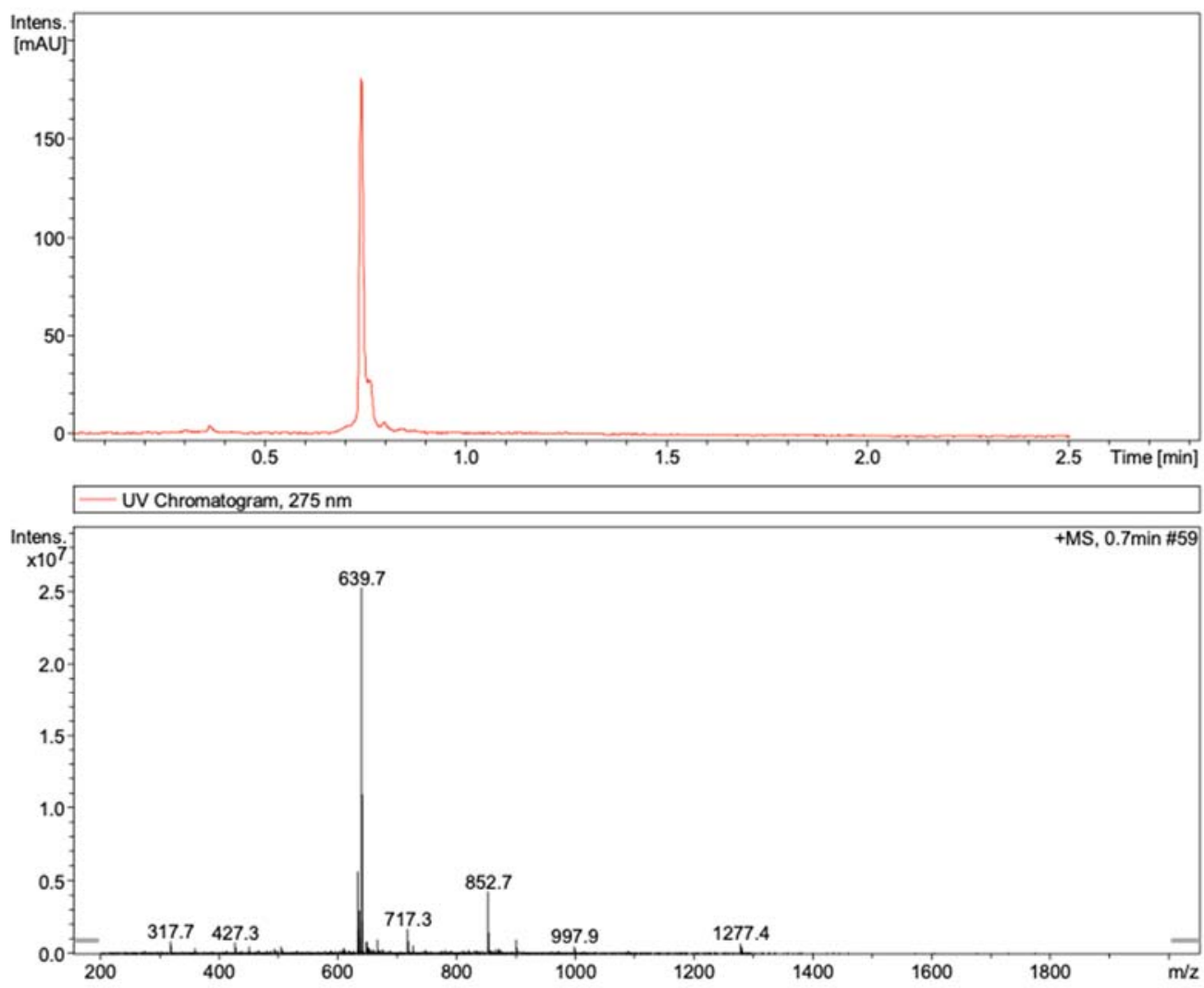

gaQEx5522 \#32-43 RT: $0.38-0.48$ AV: 6 SB: 21 0.04-0.24, 0.73-0.97 NL: $2.85 E 7$ T: FTMS + p ESI Full ms [200.00-3000.00]

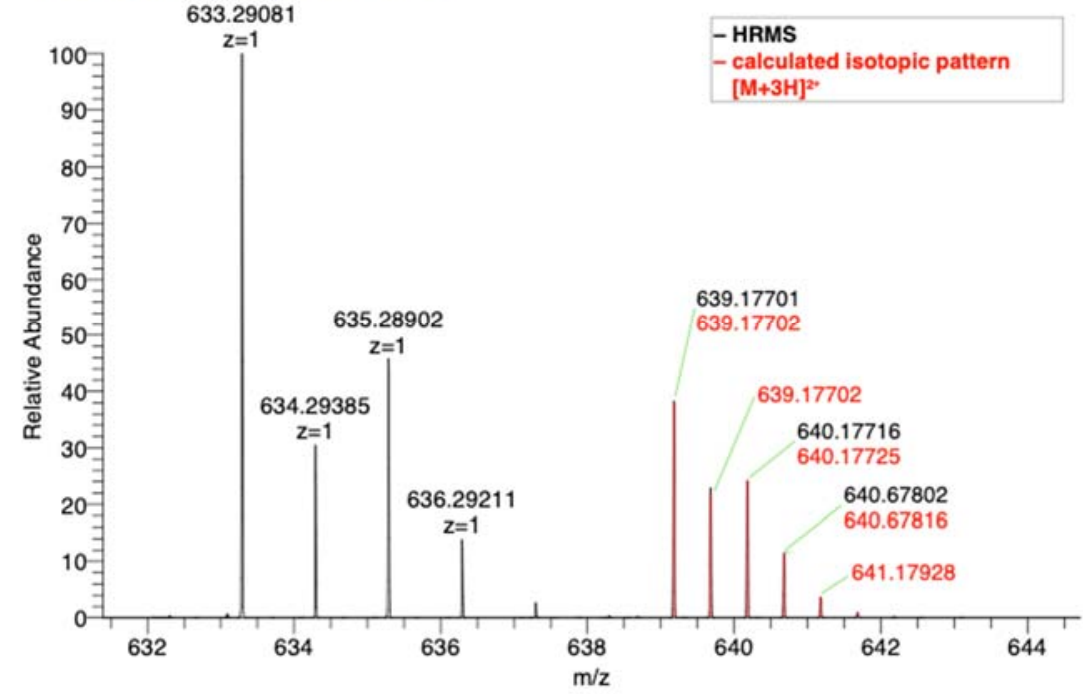

Figure S8. UPLC-MS and HR-MS of TCS2 


\subsubsection{Absorption Spectroscopy}

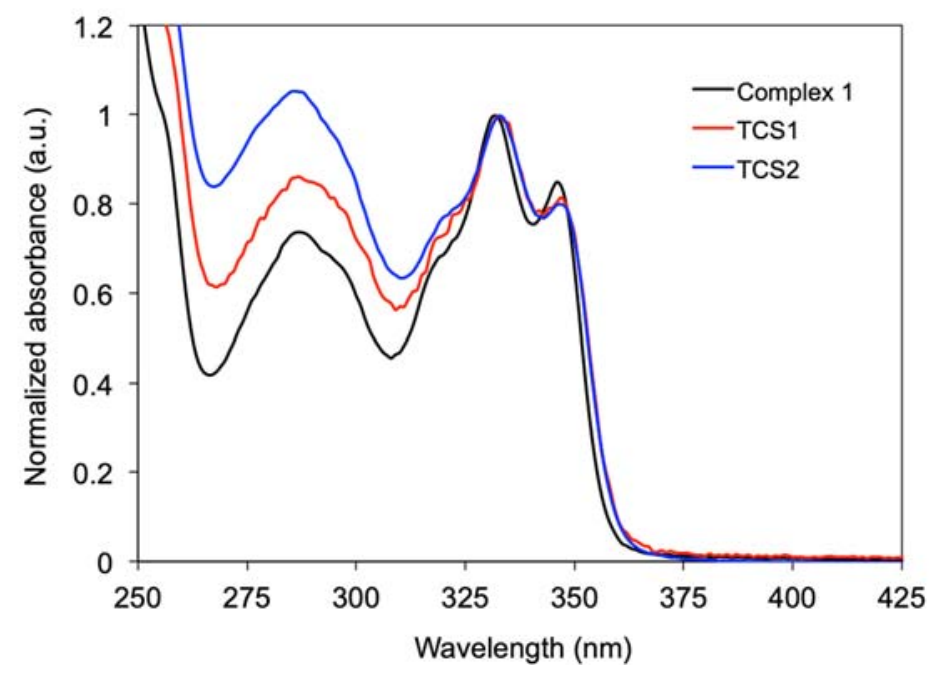

Figure S9. Normalized absorbance spectra of 1, TCS1 and TCS2 in $\mathrm{H}_{2} \mathrm{O}$.

\subsubsection{Luminescence Spectroscopy}

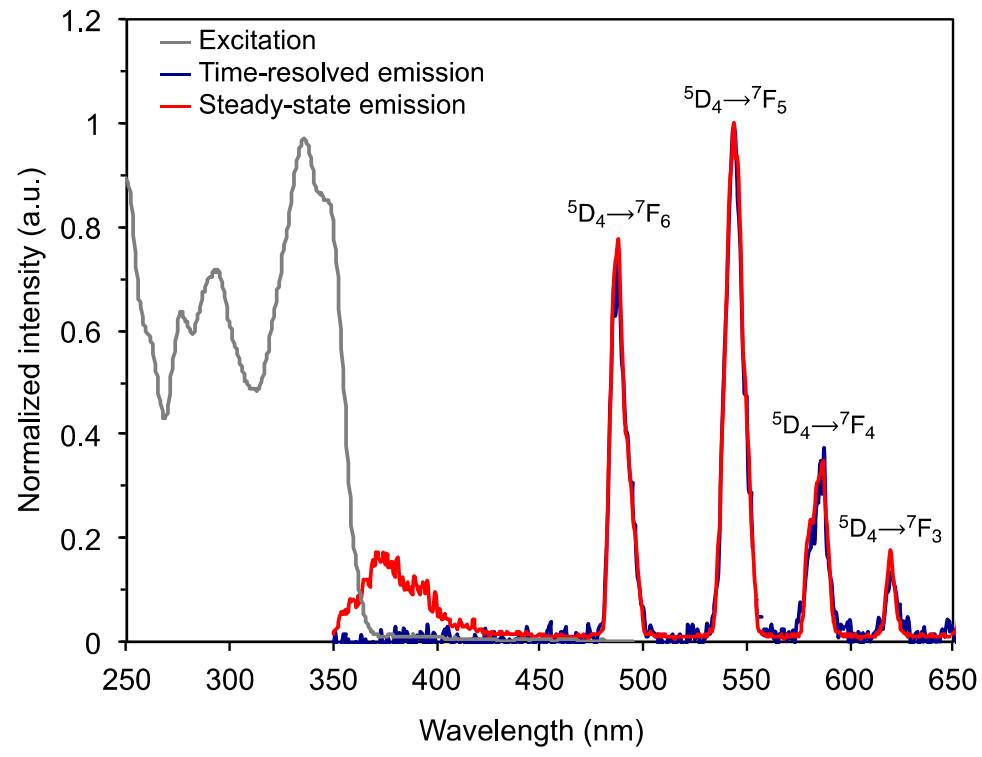

Figure S10. Excitation spectrum $\left(\lambda_{\mathrm{em}}=545 \mathrm{~nm}\right)$, time-resolved $\left(\mathrm{t}_{\text {delay }}=0.1 \mathrm{~ms}\right)$ and steadystate emission spectra $\left(\lambda_{\mathrm{exc}}=330 \mathrm{~nm}\right)$ of TCS1 in $\mathrm{H}_{2} \mathrm{O}$ at r.t. Excitation at $350 \mathrm{~nm}$ instead of $330 \mathrm{~nm}$ reduces the emission signal by about $20-30 \%$. 


\subsubsection{IR Spectroscopy}
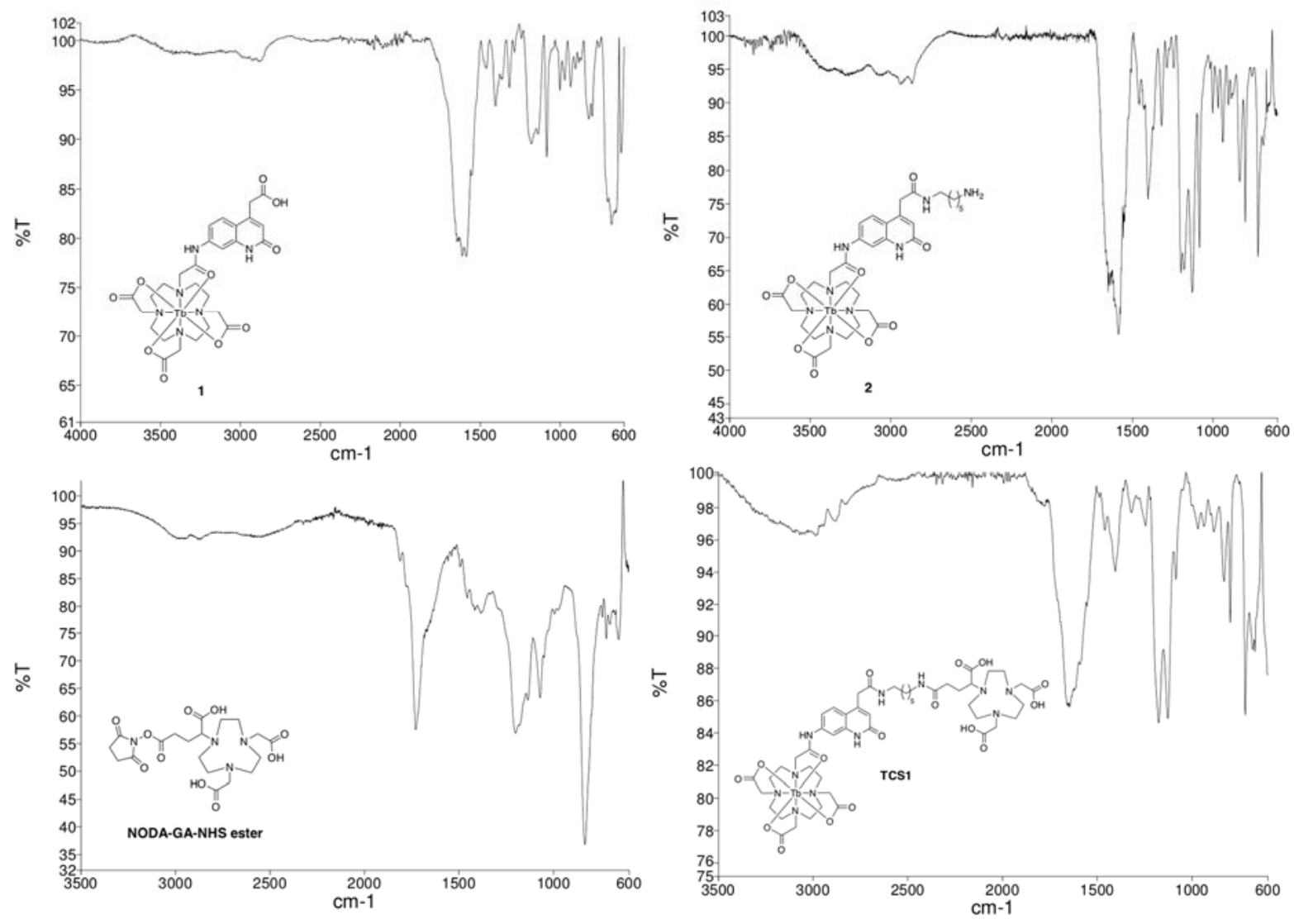

Figure S11. IR spectra (neat, ATR mode) of 1, 2, TCS1 and NODA-GA-NHS-ester 


\subsubsection{Luminescence Lifetime}

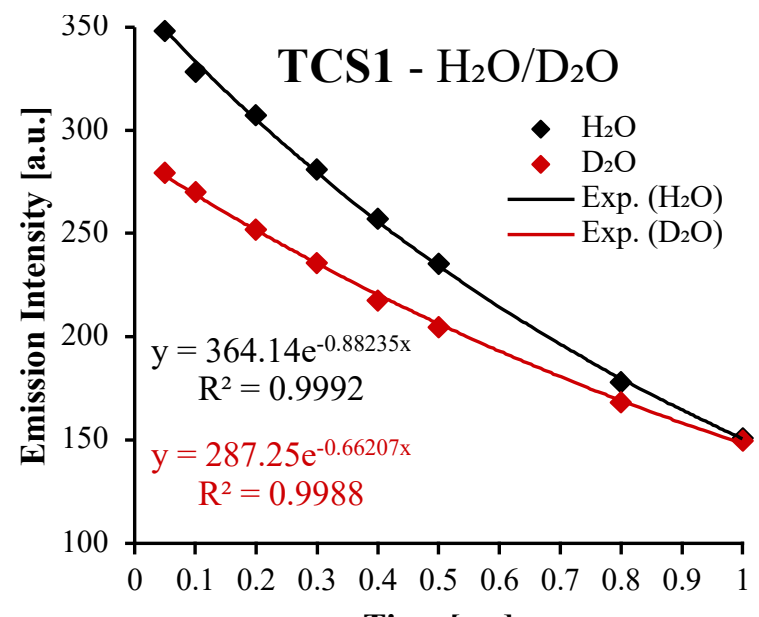

Time [ms]

$\tau_{\mathrm{H}_{2} \mathrm{O}}=0.88235^{-1} \mathrm{~ms}=\mathbf{1 . 1 3} \mathbf{~ m s}$

$\tau_{\mathrm{D}_{2} \mathrm{O}}=0.66207^{-1} \mathrm{~ms}=\mathbf{1 . 5 1} \mathbf{~ m s}$
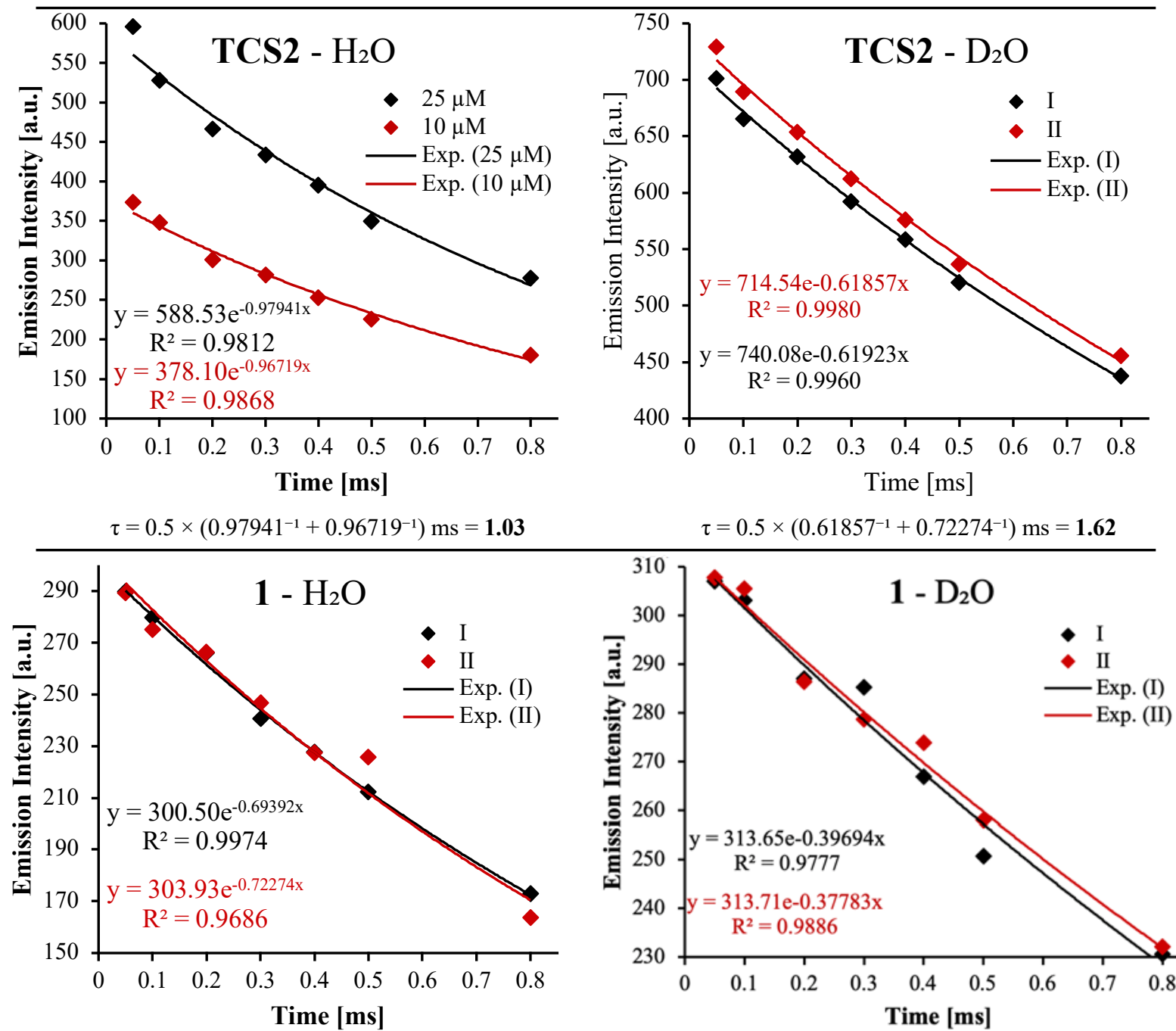

$\tau=0.5 \times\left(0.69392^{-1}+0.72274^{-1}\right) \mathrm{ms}=1.41 \mathrm{~ms}$

$\tau=0.5 \times\left(0.39694^{-1}+0.37783^{-1}\right) \mathrm{ms}=\mathbf{2 . 5 8} \mathbf{~ m s}$

Figure S12. Luminescence lifetime measurements and exponential fits for TCS1 (top), TCS2 (middle) and 1 (bottom) measured in $\mathrm{H}_{2} \mathrm{O}$ and $\mathrm{D}_{2} \mathrm{O}$ at $545 \mathrm{~nm}\left(\lambda_{\mathrm{ex}}=330 \mathrm{~nm}\right)$. 


\section{Experimental Procedures}

\subsection{Metal Ion Titrations}

Spectroscopic studies were performed in spectroscopic grade solvents using quartz cells with $1 \mathrm{~cm}$ optical pathlength at r.t. on a Perkin Elmer LS50B luminescence spectrometer. For the metal ion titrations, small volumes of stock solutions of the metal ion chloride salt in $\mathrm{H}_{2} \mathrm{O}$ were added to an aqueous solution of TCS1 $(2 \mu \mathrm{M})$ and the changes in emission spectra were monitored ( $\lambda_{\mathrm{ex}}=330 \mathrm{~nm}$; slit widths: Ex $5 \mathrm{~nm}$, Em $5 \mathrm{~nm}$; $\left.\mathrm{t}_{\text {delay }}=0 \mathrm{~ms}\right)$. The luminescent signal was found to stabilize almost immediately after addition of the metal ion, which is a desirable feature from the viewpoint of developing a rapid assay.

\subsection{Copper Sulfide Precipitation}

In order to evaluate the stability of the copper complex TCS2, precipitation of the $\mathrm{Cu}^{2+}$ ions in the form of $\mathrm{CuS}\left(K_{\mathrm{SP}}=6.3 \times 10^{-36} \mathrm{~mol}^{2} \mathrm{dm}^{-6}\right)$ was tested. For this, NaHS was added to a solution of TCS2 in $\mathrm{H}_{2} \mathrm{O}(2 \mu \mathrm{M}, \mathrm{pH} \approx 7)$ at r.t. and changes in the luminescence intensity at $545 \mathrm{~nm}$ $\left(\lambda_{\mathrm{ex}}=330 \mathrm{~nm}\right)$ were monitored (NaHS exists in the form of an approximately equal ratio of $\mathrm{HS}^{-}$and $\mathrm{H}_{2} \mathrm{~S}$ at $\mathrm{pH} 7$ ). No increase in the luminescence intensity of TCS2 could be observed upon exposure to sulfide $(1 \mu \mathrm{M}, 10 \mu \mathrm{M}, 50 \mu \mathrm{M})$, even when used in large excess with concentrations of up to $1 \mathrm{mM}$.

\subsection{Error Calculation on Hydration Numbers}

The hydration numbers $(q)$ were calculated from the difference in lifetimes in $\mathrm{H}_{2} \mathrm{O}$ and $\mathrm{D}_{2} \mathrm{O}$ using $q=5\left(\tau_{\mathrm{H}_{2} \mathrm{O}^{-1}}-\tau_{\mathrm{D}_{2} \mathrm{O}^{-1}}-0.06\right)$. The errors given on the $q$ values $\left(\mathrm{s}_{q}\right)$ were calculated based on the error in the regression of the lifetime measurements $(\mathrm{s} \tau)$ using error propagation theory as given by:

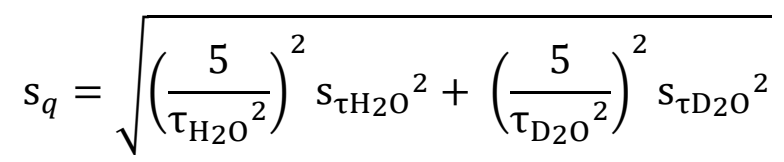


Table S1. Errors in lifetime measurements and calculated errors in hydration numbers.

\begin{tabular}{ccccccc}
\hline & $\begin{array}{c}\tau_{\mathrm{H}_{2} \mathrm{O}} \\
(\mathrm{ms})\end{array}$ & $\begin{array}{c}\text { Error }^{[\mathrm{a}]} \\
\mathrm{s} \tau\end{array}$ & $\begin{array}{c}\tau_{\mathrm{D}_{2} \mathrm{O}} \\
(\mathrm{ms})\end{array}$ & $\begin{array}{c}\text { Error } \\
{ }^{[\mathrm{a}]}\end{array}$ & $q$ & $\begin{array}{c}\text { Error on } \boldsymbol{q} \\
\mathbf{s} \tau\end{array}$ \\
\hline $\mathbf{1}$ & 1.41 & 0.113 & 2.58 & 0.181 & 1.3 & $\mathbf{0 . 3 1}$ \\
$\mathbf{T C S 1}$ & 1.13 & 0.0113 & 1.51 & 0.0151 & 0.8 & $\mathbf{0 . 0 5 5}$ \\
$\mathbf{T C S 2}$ & 1.03 & 0.0618 & 1.62 & 0.0486 & 1.5 & $\mathbf{0 . 3 1}$ \\
\hline
\end{tabular}

[a] The errors of the regression (Figure S12).

\section{Detection Range and LOD of Small Molecule $\mathrm{Cu}^{2+}$ Sensors}

Table S2. Comparison of probes for the detection of $\mathrm{Cu}^{2+}$ ions.

\begin{tabular}{|c|c|c|c|c|c|}
\hline Sensor & Method & System & $\begin{array}{c}\text { Detection Range } \\
(\mu \mathrm{M})\end{array}$ & $\begin{array}{l}\text { LOD } \\
(\mu \mathrm{M})\end{array}$ & Reference \\
\hline $\mathrm{S} 1$ & Ln luminescence & Tb-complex, turn-off & $\sim 5.0-20$ & - & Gunnlaugson et al. ${ }^{3}$ \\
\hline $\mathrm{S} 2$ & Ln luminescence & Eu-complex, turn-off & $\sim 0.1-0.7$ & - & Liang et al. ${ }^{4}$ \\
\hline S6 & Ln luminescence & Eu-complex, turn-off & - & 9.6 & Aulsebrook et al. ${ }^{5}$ \\
\hline S7 & Ln luminescence & Eu-complex, turn-off & - & 1 & Aulsebrook et al. ${ }^{5}$ \\
\hline S8 & Ln luminescence & Eu-complex, turn-off & - & 0.0037 & Aulsebrook et al. ${ }^{5}$ \\
\hline S9 & Fluorescence & Coumarin derivative & $0.1-1$ & 0.015 & Chen et al. ${ }^{6}$ \\
\hline $\mathrm{S} 10$ & Fluorescence & Acridine derivative & $1-16$ & 0.12 & Dai et al. ${ }^{7}$ \\
\hline
\end{tabular}

\section{Copper Concentrations in Biological Samples}

Plasma copper levels for healthy adults are $\sim 10 \mu \mathrm{M}$ (for copper level associated disease, such as Menke's, 2-6 $\mu \mathrm{M}) .{ }^{8}$ The non-ceruloplasmin-bound copper ("free copper") concentration is much lower. Salivary copper levels are $\sim 1 \mu \mathrm{M} .{ }^{8}$ For TCS1 $\left(2 \mu \mathrm{M}\right.$ in $\left.\mathrm{H}_{2} \mathrm{O}\right)$, a linear relationship between the luminescence intensity and the $\mathrm{Cu}^{2+}$ ion concentration could be obtained in the concentration window of $0.1-1.5 \mu \mathrm{M}$. 


\section{References}

(1) Jenie, S. N. A.; Du, Z.; McInnes, S. J. P.; Ung, P.; Graham, B.; Plush, S. E.; Voelcker, N. H. Biomolecule Detection In Porous Silicon Based Microcavities Via Europium Luminescence Enhancement. J. Mater. Chem. B 2014, 2, 7694-7703.

(2) Reddy, D. R.; Pedro Rosa, L. E.; Miller, L. W. Luminescent Trimethoprim-Polyaminocarboxylate Lanthanide Complex Conjugates For Selective Protein Labeling And Time-Resolved Bioassays. Bioconjuage Chem. 2011, 22, 1402-1409.

(3) McMahon, B. K.; Gunnlaugsson, T. Lanthanide Luminescence Sensing Of Copper And Mercury Ions Using An Iminodiacetate-Based Tb( III )-Cyclen Chemosensor. Tetrahedron Lett. 2010, 51, 5406-5410.

(4) Liang, Z.; Tsoi, T.-H.; Chan, C.-F.; Dai, L.; Wu, Y.; Du, G.; Zhu, L.; Lee, C.-S.; Wong, W.-T.; Law, G.L.; Wong, K.-L. A Smart “Off-On” Gate For The In Situ Detection Of Hydrogen Sulphide With Cu( II )Assisted Europium Emission. Chem. Sci. 2016, 7, 2151-2156.

(5) Aulsebrook, M. L.; Graham, B.; Grace, M. R.; Tuck, K. L. Lanthanide Complexes For LuminescenceBased Sensing Of Low Molecular Weight Analytes. Coord. Chem. Rev. 2018, 375, 191-220.

(6) Chen, H.; Jia, S.; Zhang, J.; Jang, M.; Chen, X.; Koh, K.; Wang, Z. Sensitive Detection Of Copper(II) Ions Based On The Conformational Change Of Peptides By Surface Plasmon Resonance Spectroscopy. Anal. Methods 2015, 7, 8942-8946.

(7) Dai, Q.; Liu, H.; Gao, C.; Li, W.; Zhu, C.; Lin, C.; Tan, Y.; Yuan, Z.; Jiang, Y. A One-Step Synthesized Acridine-Based Fluorescent Chemosensor For Selective Detection Of Copper (II) Ions And Living Cell Imaging. New J. Chem. 2018, 42, 613-618.

(8) Brody, T. Inorganic Nutrients. In Nutritional Biochemistry; Academic Press: San Diego, 1999; pp 693878. 